\title{
TRAVELING WAVES AND SHOCKS IN A VISCOELASTIC GENERALIZATION OF BURGERS' EQUATION
}

\author{
VICTOR CAMACHO*, ROBERT D. GUY ${ }^{\dagger}$, AND JON JACOBSEN ${ }^{\ddagger}$
}

\begin{abstract}
We consider traveling wave phenomena for a viscoelastic generalization of Burgers' equation. For asymptotically constant velocity profiles we find three classes of solutions corresponding to smooth traveling waves, piecewise smooth waves, and piecewise constant (shock) solutions. Each solution type is possible for a given pair of asymptotic limits and we characterize the dynamics in terms of the relaxation time and viscosity.
\end{abstract}

Key words. non-Newtonian fluids, fast-slow dynamics, vanishing viscosity solutions

AMS subject classifications. 35L67, 76A10, 35Q53

1. Introduction. Burgers' equation

$$
u_{t}+u u_{x}=\epsilon u_{x x}
$$

is perhaps the simplest model that couples the nonlinear convective behavior of fluids with the dissipative viscous behavior. Introduced by Burgers [5] as a model for turbulence, equation (1.1) and its inviscid counterpart

$$
u_{t}+u u_{x}=0,
$$

are essential for their role in modeling a wide array of physical systems such as traffic flow, shallow water waves, and gas dynamics $[17,18,19,23]$. The equations also provide fundamental pedagogical examples for many important topics in nonlinear PDE such as traveling waves, shock formation, similarity solutions, singular perturbation, and numerical methods for parabolic and hyperbolic equations (see e.g., $[9,14,20,23]$ ).

The parabolic equation (1.1) has the property that smooth initial data yields smooth solutions for all $t>0$. In contrast, smooth initial data for the hyperbolic equation (1.2) can develop jump discontinuities in finite time (shock formation). One technique for studying shock wave solutions of (1.2) is to study smooth traveling wave solutions of (1.1) in the limit as $\epsilon \rightarrow 0$.

In this paper we consider how the addition of viscoelasticity affects traveling wave solutions of Burgers' equation. The equations we consider are

$$
\begin{aligned}
u_{t}+u u_{x} & =\sigma_{x} \\
\sigma_{t}+u \sigma_{x}-\sigma u_{x} & =\alpha u_{x}-\beta \sigma .
\end{aligned}
$$

The constitutive law (1.4) resembles a one-dimensional version of the upper convected Maxwell model [11]. The relaxation time is $\lambda=\beta^{-1}$, and $\alpha=\mu \lambda^{-1}$ can be interpreted as the elastic modulus of the material if there were no relaxation of stress $(\beta=0)$. In

*Mathematics Department, Harvey Mudd College, 301 Platt Blvd., Claremont, CA, 91711, (vcamacho@hmc.edu). The research of this author was supported by a grant from the Baker Foundation.

${ }^{\dagger}$ Mathematics Department, University of California Davis, Davis CA, 95616, (guy@math.ucdavis.edu). The research of this author was supported in part by NSF grants DMS-0139926 and DMS-0540779.

${ }_{\ddagger}^{\ddagger}$ Mathematics Department, Harvey Mudd College, 301 Platt Blvd., Claremont, CA, 91711, (jacobsen@math.hmc.edu). The research of this author was supported by a grant from the Beckman Research Program at Harvey Mudd College. 
the other limit of instantaneous relaxation of stress $(\lambda \rightarrow 0)$, equation (1.4) reduces to $\sigma=\mu u_{x}$, and the system (1.3)-(1.4) is equivalent to Burgers' equation (1.1) with fluid viscosity $\mu=\epsilon$.

The remainder of the paper is organized as follows. In Section 2 we give a brief introduction to viscoelastic fluids and explain the reduction and constitutive law for our model. We show in Section 3 that traveling wave solutions to (1.3)-(1.4) exist only when the viscosity (or elastic modulus) is above a certain threshold. As the viscosity approaches this threshold, singularities in the derivative appear, and numerical experiments suggest that shocks develop when the viscosity is below threshold. The system (1.3)-(1.4) is nonconservative, and therefore the classical theory for systems of conservation laws (cf. $[9,23]$ ) cannot be used to analyze singular solutions. A generalized theory of weak solutions to nonconservative hyperbolic equations has been developed for such problems $[2,7,8]$.

We take a different approach and analyze the shock solutions by introducing an additional viscosity to regularize the problem. Using singular perturbation theory, we show in Section 4 that traveling waves exist for all parameters in the regularized problem, and the waves limit to shock solutions as the additional viscosity goes to zero. This method of vanishing viscosity is a well-known technique for analyzing weak solutions of nonconservative hyperbolic equations, such as the Hamilton-Jacobi equations [9]. Finally, in Section 5 we discuss the effect of different parameters on the solution structure, how the results depend on the choice of one-dimensional reduction, and a possible application of the results to numerical methods for viscoelastic flows.

2. Viscoelastic Fluids. In this section we discuss how the constitutive law in (1.4) is related to a standard constitutive law for viscoelastic fluids. The discussion here is not meant to be extensive. For more comprehensive treatments of viscoelastic fluids, see $[3,4,11,12]$.

The incompressible Navier-Stokes equations are

$$
\begin{aligned}
\rho\left(\boldsymbol{u}_{t}+\boldsymbol{u} \cdot \nabla \boldsymbol{u}\right) & =-\nabla p+\mu \Delta \boldsymbol{u} \\
\nabla \cdot \boldsymbol{u} & =0 .
\end{aligned}
$$

The momentum equation (2.1), can be expressed as

$$
\rho\left(\boldsymbol{u}_{t}+\boldsymbol{u} \cdot \nabla \boldsymbol{u}\right)=-\nabla p+\nabla \cdot \boldsymbol{\sigma}_{\mathbf{v}}
$$

where the (Newtonian) viscous stress $\boldsymbol{\sigma}_{\mathbf{v}}$ is defined by

$$
\boldsymbol{\sigma}_{\mathbf{v}}=2 \mu \boldsymbol{D}=\mu\left(\nabla \boldsymbol{u}+\nabla \boldsymbol{u}^{\mathrm{T}}\right)
$$

This Newtonian constitutive law means that the fluid stress is proportional to the deformation rate tensor. In contrast, the stress in viscoelastic fluids includes some time history of the deformation.

One of the simplest constitutive laws for viscoelastic materials is the Maxwell model. Consider a linear spring and dashpot in series, with spring constant $k$ and damping coefficient $\mu$. The stress, $\sigma$, in the element is

$$
\lambda \dot{\sigma}+\sigma=\mu \dot{\epsilon}
$$

where $\epsilon$ is the strain in the element, and $\lambda=k / \mu$ is the relaxation time. The linear Maxwell model for a continuum is

$$
\lambda \boldsymbol{\sigma}_{t}+\boldsymbol{\sigma}=2 \mu \boldsymbol{D}
$$


However, this is not a valid constitutive law because it is not frame invariant [11]. That is, the stress depends on the reference frame. Frame invariance is achieved by choosing an appropriate time derivative, akin to the material derivative for the velocity field. One frame invariant time derivative is the upper convected derivative, defined by

$$
\stackrel{\nabla}{\boldsymbol{S}}=\boldsymbol{S}_{t}+\boldsymbol{u} \cdot \nabla \boldsymbol{S}-\nabla \boldsymbol{u} \boldsymbol{S}-\boldsymbol{S} \nabla \boldsymbol{u}^{\mathrm{T}}
$$

Replacing the partial time derivative in (2.6) with the upper convected derivative gives the Upper Convected Maxwell (UCM) equation

$$
\lambda \stackrel{\nabla}{\sigma}+\boldsymbol{\sigma}=2 \mu \boldsymbol{D} .
$$

The $i j$ component in (2.8) satisfies

$$
\lambda\left(\frac{\partial \sigma_{i j}}{\partial t}+u_{k} \frac{\partial \sigma_{i j}}{\partial x_{k}}-\frac{\partial u_{i}}{\partial x_{k}} \sigma_{k j}-\sigma_{i k} \frac{\partial u_{j}}{\partial x_{k}}\right)+\sigma_{i j}=\mu\left(\frac{\partial u_{i}}{\partial x_{j}}+\frac{\partial u_{j}}{\partial x_{i}}\right),
$$

where summation is over the repeated index $k$. Although there are many other frame invariant derivatives, in this paper we consider a one-dimensional reduction, in which case they yield identical reductions.

A one-dimensional version of the UCM equation is

$$
\lambda\left(\sigma_{t}+u \sigma_{x}-\sigma u_{x}\right)+\sigma=\mu u_{x} .
$$

However, there are other reasonable choices for a one-dimensional UCM equation. For example, the equation for $\sigma_{11}$ when $\boldsymbol{u}=\left(u_{1}, 0,0\right)$ is

$$
\lambda\left(\sigma_{t}+u \sigma_{x}-2 \sigma u_{x}\right)+\sigma=2 \mu u_{x}
$$

where we have dropped the subscripts on the stress and velocity. The upper convected derivative must be used in (2.8) because this is the time derivative of a tensor in a moving continuum. In one-dimension, the stress is a scalar, so it would also be reasonable to simply use the material derivative for the time derivative. In this case the constitutive law is

$$
\lambda\left(\sigma_{t}+u \sigma_{x}\right)+\sigma=\mu u_{x} .
$$

In this paper we analyze the first UCM equation (2.10). While all three models have similar results, equation (2.10) is more robust, in that all of the phenomena that occur in (2.11) and (2.12), also occur in (2.10). In Section 5 we discuss how the results change if (2.11) or (2.12) is used instead.

Equation (2.10) is equivalent to (1.4). This is seen by dividing through by the relaxation time $\lambda$ to get

$$
\sigma_{t}+u \sigma_{x}-\sigma u_{x}=\alpha u_{x}-\beta \sigma
$$

where

$$
\begin{aligned}
& \alpha=\mu \lambda^{-1}, \\
& \beta=\lambda^{-1} .
\end{aligned}
$$


The parameter $\alpha$ can be interpreted as the elastic modulus of the material if there were no relaxation of stress $(\beta=0)$. It is somewhat arbitrary whether the constitutive law is expressed in terms of the relaxation time $(\lambda)$ and viscosity $(\mu)$ or elastic modulus $(\alpha)$ and decay rate $(\beta)$. In this paper we primarily use the later, but sometimes we express results using both sets of parameters for additional insight.

In Section 4 we consider a modification to the Maxwell constitutive law (1.4). We include a second viscous term, one without memory, so that the system becomes

$$
\begin{gathered}
u_{t}+u u_{x}=\sigma_{x}+\epsilon u_{x x} \\
\sigma_{t}+u \sigma_{x}-\sigma u_{x}=\alpha u_{x}-\beta \sigma .
\end{gathered}
$$

The addition of the second viscous term can be considered as a one-dimensional version of the Oldroyd-B constitutive law [12].

We note that the one-dimensional constitutive law studied in this paper is not a physical reduction from the three dimensional UCM. It is a reduction in the same sense that Burgers equation is a reduction. One may wonder what, if any, physical significance there is to the problem that we analyze in this paper. Using high-resolution Godunov schemes for the advection terms in the Navier-Stokes equations requires solving Burgers equation [1]. Analogously, systems of the form (2.10) and (2.11) arise in the application of wave propagation schemes to viscoelastic fluids [10, 22]. This was the original inspiration for this study, but not the sole motivation. It it interesting to explore what happens to traveling waves in Burgers equation (1.1) if the viscous term is replaced by a viscoelastic term, and the most natural starting point is the Maxwell model. Thus the one dimensional constitutive laws considered were chosen to resemble the UCM equation.

3. Traveling Waves. To find traveling wave solutions to (1.3)-(1.4) we consider solutions of the form $u(x, t)=U(\xi)$ and $\sigma(x, t)=S(\xi)$, where $\xi=x-c t$ for some constant $c$. In traveling wave coordinates, the system is

$$
\begin{aligned}
-c U^{\prime}+U U^{\prime} & =S^{\prime} \\
-c S^{\prime}+U S^{\prime}-S U^{\prime} & =\alpha U^{\prime}-\beta S .
\end{aligned}
$$

We consider traveling waves that correspond to heteroclinic connections between two equilibrium points with given velocity values at infinity. The equilibrium points of the system correspond to all states with $S=0$, thus we assume the following asymptotic boundary conditions:

$$
\begin{array}{cl}
U(-\infty)=u_{\ell}, & S(-\infty)=0 \\
U(\infty)=u_{r}, & S(\infty)=0 .
\end{array}
$$

In the next section we examine for which values of $u_{\ell}, u_{r}, \alpha$, and $\beta$ do solutions of this problem exist.

3.1. Existence. Integrating equation (3.1) gives the stress in terms of the velocity as

$$
S=\frac{U^{2}}{2}-c U+A
$$


where $A$ is the integration constant. Applying the boundary conditions, the wave speed and integration constant are

$$
\begin{aligned}
A & =\frac{u_{\ell} u_{r}}{2} \\
c & =\frac{u_{\ell}+u_{r}}{2} .
\end{aligned}
$$

Note that if a traveling wave exists, then it moves with the same speed as traveling waves in Burgers' equation (1.1) and shock waves in inviscid Burgers' equation (1.2).

We obtain the equation for the velocity profile, $U$, by using (3.1) and (3.5) to eliminate $S$ and $S^{\prime}$ in (3.2) to get

$$
U^{\prime}=\frac{-\beta(U(U / 2-c)+A)}{U(U / 2-c)+c^{2}-A-\alpha} .
$$

Using (3.6) and (3.7), this simplifies to

$$
U^{\prime}=\frac{-\beta\left(U-u_{\ell}\right)\left(U-u_{r}\right)}{\left(U-u_{\ell}\right)\left(U-u_{r}\right)+2\left(\left(\frac{u_{\ell}-u_{r}}{2}\right)^{2}-\alpha\right)} .
$$

From the dynamics of this equation we extract conditions for the existence of traveling waves. The two equilibrium points are clearly $U=u_{\ell}$ and $U=u_{r}$, and a traveling wave corresponds to a one-dimensional flow from one equilibrium point to the other. There are two cases to consider: $u_{\ell}>u_{r}$ and $u_{\ell}<u_{r}$.

First we suppose that $u_{\ell}>u_{r}$. For a traveling wave to exist, we need that $U^{\prime}<0$ for $U \in\left(u_{r}, u_{\ell}\right)$. The numerator of (3.9) is positive in this interval. The maximum value of $\left(U-u_{\ell}\right)\left(U-u_{r}\right)$ is 0 , and so the denominator is always negative provided $\left(\left(u_{\ell}-u_{r}\right) / 2\right)^{2}-\alpha<0$, in which case $U^{\prime}<0$ for $U \in\left(u_{r}, u_{\ell}\right)$.

Next, consider the case $u_{\ell}<u_{r}$. A traveling wave exists if $U^{\prime}>0$ for $U \in\left(u_{\ell}, u_{r}\right)$. As before, the numerator of (3.9) is positive for $U \in\left(u_{\ell}, u_{r}\right)$, and thus we examine the sign of the denominator. The minimum value of $\left(U-u_{\ell}\right)\left(U-u_{r}\right)$ is $-\left(\left(u_{\ell}-u_{r}\right) / 2\right)^{2}$, in which case it follows $U^{\prime}>0$ provided $\left(\left(u_{\ell}-u_{r}\right) / 2\right)^{2}-2 \alpha>0$.

Combining these two cases, we have the following result: a traveling wave solution to (1.3)-(1.4) with boundary conditions (3.3)-(3.4) exists if and only if

$$
u_{\ell}>u_{r} \quad \text { and } \quad \alpha>\left(\frac{u_{\ell}-u_{r}}{2}\right)^{2},
$$

or

$$
u_{\ell}<u_{r} \quad \text { and } \quad 2 \alpha<\left(\frac{u_{\ell}-u_{r}}{2}\right)^{2} .
$$

Equivalently, no traveling wave solutions exist if

$$
\frac{\left(u_{\ell}-u_{r}\right)^{2}}{8} \leq \alpha \leq \frac{\left(u_{\ell}-u_{r}\right)^{2}}{4} .
$$

Using (2.14) to express this condition in terms of the relaxation time and viscosity, we see that no traveling wave solution exists if

$$
\frac{\left(u_{\ell}-u_{r}\right)^{2}}{8} \leq \frac{\mu}{\lambda} \leq \frac{\left(u_{\ell}-u_{r}\right)^{2}}{4}
$$


In comparison, for viscous Burgers' equation (1.1), traveling waves with $u_{\ell}>u_{r}$ exist for any positive viscosity. By adding elasticity we see that, for a fixed relaxation time $\lambda$, there is now a minimal viscosity required for such waves to exist. In the following sections we explore what happens to these wave solutions when the viscosity is reduced beyond this minimal viscosity.

3.2. Wave Profile. The shape of the wave is found by integrating (3.9). The solution is

$$
\beta\left(\xi-\xi_{0}\right)=\frac{2\left(\left(\frac{u_{\ell}-u_{r}}{2}\right)^{2}-\alpha\right)}{u_{\ell}-u_{r}} \log \left|\frac{U(\xi)-u_{r}}{U(\xi)-u_{\ell}}\right|-U(\xi) .
$$

When a traveling wave exists, the profile is defined implicitly by (3.14). However, when a traveling wave fails to exist, we can still plot the implicit solutions of (3.14). In Figure 3.1 we plot the curve defined by (3.14) for four different value of $\alpha$, while keeping the other parameter values fixed at $u_{\ell}=2, u_{r}=0$, and $\beta=1$. For these parameter values, a traveling wave exists when $\alpha>1$. In Figure 3.1(a) the wave profile is shown for $\alpha=1.2$. As $\alpha$ approaches 1 , the wave profile approaches the piecewise linear function shown in Figure 3.1(b). As $\alpha$ is decreased further, the curve becomes multivalued and the asymptotic values are no longer satisfied. Figure 3.1(c) shows the solution for $\alpha=0.9$. As $\alpha$ decreases even further, the solution of (3.14) returns to being single-valued, but no longer yields a traveling wave solution with the given asymptotic limits. This transition occurs at $\alpha=\frac{1}{2}\left(\frac{u_{\ell}-u_{r}}{2}\right)^{2}$, which corresponds to when $U^{\prime}$ returns to being one-signed (now positive), corresponding to the lower limit of equation (3.13). Figure 3.1(d) shows the solution for $\alpha=0.25$.

3.3. Numerical Simulations. In this section we consider numerical simulations of the full PDE system (1.3)-(1.4). According to (3.10), when $u_{\ell}>u_{r}$ there is a minimal viscosity in order for traveling waves to exist. In numerical simulations of this case, these traveling wave solutions appear to be stable and travel with the speed $c=\left(u_{\ell}+u_{r}\right) / 2$, as in (3.7). We found that for any initial data, as long the asymptotic limits were maintained, the solution approached the traveling wave profile given by (3.14). On the other hand according to (3.11), when $u_{\ell}<u_{r}$, traveling waves exist as long as the viscosity is below a certain threshold. In simulations of the PDE system for this case, these waves did not appear to be stable, rather the solutions always rarefy. Accordingly, from this point on we consider only the stable case of $u_{\ell}>u_{r}$.

We next consider what happens when the viscosity is below the minimal value, corresponding to the implicit plots shown in Figure 3.1(c)-(d). We solve the full system (1.3)-(1.4) numerically by splitting the update at each time step into three substeps. First we take a step including only the advection terms

$$
\begin{aligned}
& u_{t}+u u_{x}=0 \\
& \sigma_{t}+u \sigma_{x}=0,
\end{aligned}
$$

and use an upwinding method. Next we take a step including the elastic terms

$$
\begin{aligned}
u_{t} & =\sigma_{x} \\
\sigma_{t}-\sigma u_{x} & =\alpha u_{x} .
\end{aligned}
$$

We linearize the $\sigma u_{x}$ term in each grid cell by treating this term as $\sigma_{j}^{n} u_{x}$ through the time step, where $\sigma_{j}^{n}$ is the value of the stress at time step $n$ at grid cell $j$. This 


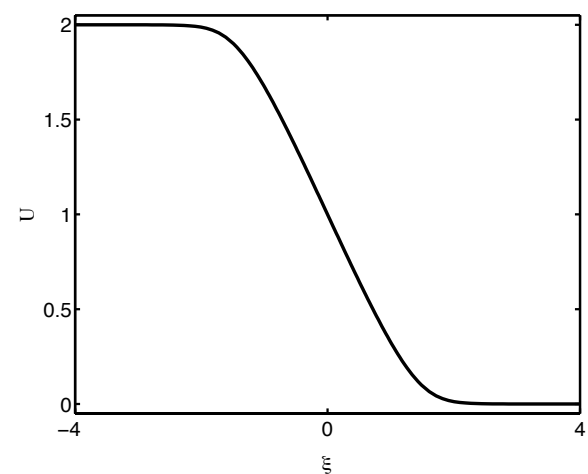

(a)

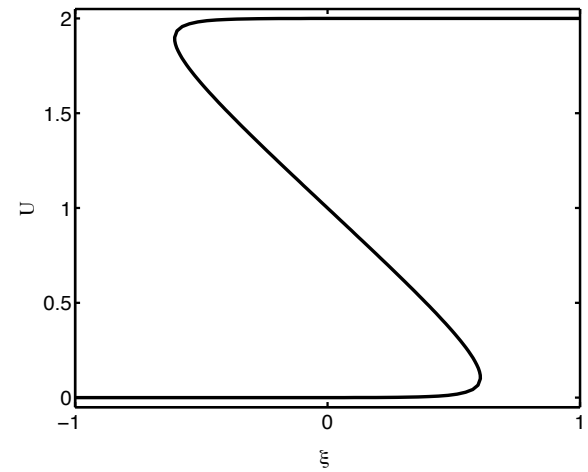

(c)

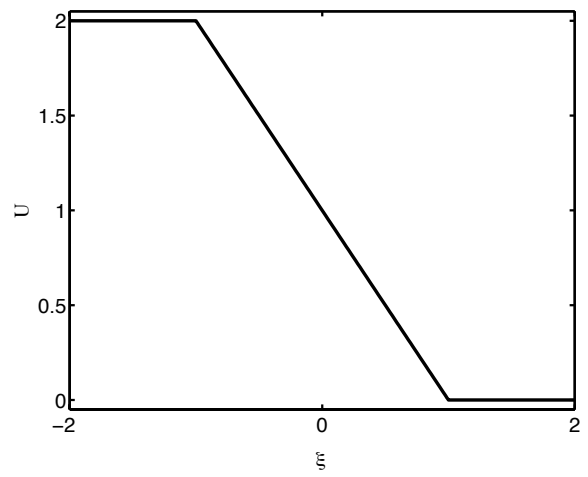

(b)

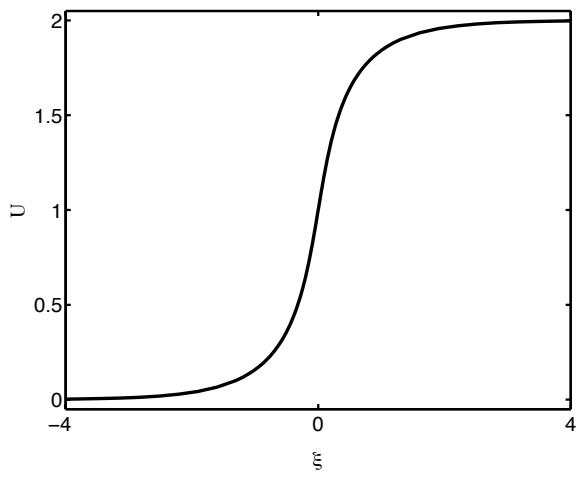

(d)

FIG. 3.1. Plots of the solution curves to (3.14). The parameters are $u_{\ell}=2, u_{r}=0, \beta=1$. Four different values of $\alpha$ are plotted: (a) $\alpha=1.2$, (b) $\alpha=1$, (c) $\alpha=0.9$, (d) $\alpha=0.25$. For these values of $u_{\ell}$ and $u_{r}$, no wave exists for $\alpha<1$.

linearized system is a variable coefficient wave equation, which we update by a wave propagation method as described in [16]. Finally, we update the stress by taking a step of

$$
\sigma_{t}=-\beta \sigma
$$

For the initial condition we set the velocity equal to the traveling wave profile corresponding to viscous Burgers' equation with a given viscosity and set the stress to zero.

As suggested by Figure 3.1(c)-(d), we find two distinct cases, corresponding to whether

$$
\frac{1}{2}\left(\frac{u_{\ell}-u_{r}}{2}\right)^{2}<\alpha<\left(\frac{u_{\ell}-u_{r}}{2}\right)^{2}
$$

or

$$
0<\alpha<\frac{1}{2}\left(\frac{u_{\ell}-u_{r}}{2}\right)^{2}
$$


In both cases we find that the solutions develop into traveling waves, however now with jump discontinuities in the wave profile. These numerical solutions propagate with the wave speed $c=\left(u_{\ell}+u_{r}\right) / 2$, the same wave speed as smooth traveling wave solutions. When $\alpha$ satisfies (3.20) the profile is piecewise smooth, with two shocks as indicated in Figure 3.2-(a). We refer to this solution as the double-shock solution. As $\alpha$ ranges between the limiting values of (3.20) the height of each jump discontinuity ranges from 0 when $\alpha=\left(\frac{u_{\ell}-u_{r}}{2}\right)^{2}$, to 1 when $\alpha=\frac{1}{2}\left(\frac{u_{\ell}-u_{r}}{2}\right)^{2}$, which yields a piecewise constant solution. This piecewise constant solution persists when $\alpha$ satisfies (3.21), as indicated in Figure 3.2-(b). This resembles a classic shock solution of the Riemann problem for the inviscid Burgers' equation.

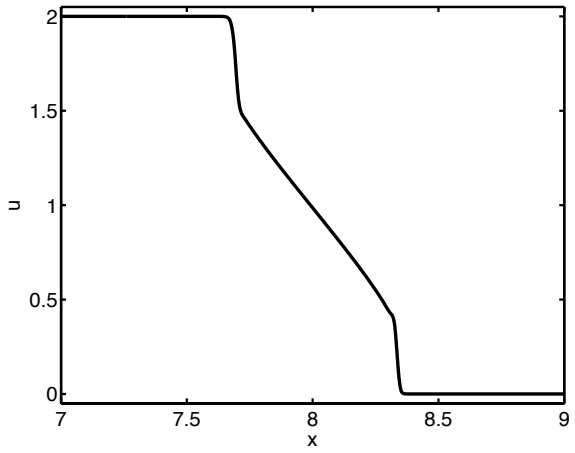

(a)

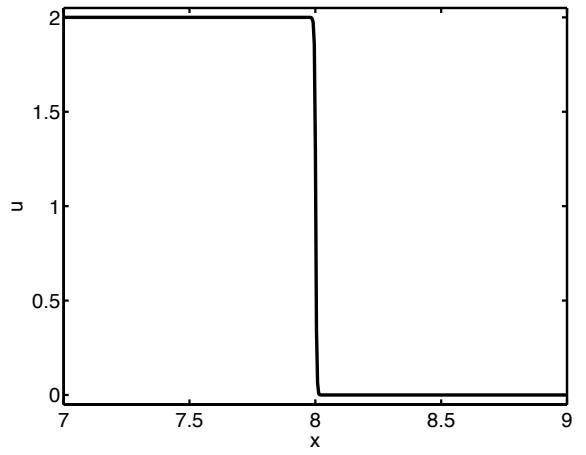

(b)

FIG. 3.2. Plots of the wave profile found by solving (1.3)-(1.4) with smooth traveling wave initial data. The simulations were run until the profile stabilized. The smooth waves develop apparent jump discontinuities, whose type depends on whether $\alpha$ satisfies (3.20) or (3.21), and travel with fixed speed. The parameter values are $u_{\ell}=2, u_{r}=0, \beta=1$ and (a) $\alpha=0.8$; (b) $\alpha=0.25$.

When solving equations with discontinuities care must be taken in order to capture the correct solution. These numerical solutions may not be the correct solutions, but they raise several questions that warrant further investigation. For example, as the PDE is not given by a system of conservation laws, what is the "correct" weak solution? In the case of the double-shock solution, what determines the shock height? What determines the shape of the solution between the two shocks? Why is that we see a double-shock solution? In the next section we answer these questions by introducing a second viscous term to regularize the equations and analyzing the system in the limit of small viscosity.

4. Vanishing Viscosity Solution. In this section we add a viscous regularization term on the velocity:

$$
\begin{gathered}
u_{t}+u u_{x}=\sigma_{x}+\epsilon u_{x x} \\
\sigma_{t}+u \sigma_{x}-\sigma u_{x}=\alpha u_{x}-\beta \sigma,
\end{gathered}
$$

for $\epsilon>0$. With the extra viscous term, this system can be viewed as a one-dimensional version of the Oldroyd-B constitutive law [12]. To study the double-shock and shock solutions of (1.3)-(1.4) we consider traveling wave solutions of this extended system in the limit $\epsilon \rightarrow 0$. 
In traveling wave coordinates, the system becomes

$$
\begin{aligned}
-c U^{\prime}+U U^{\prime} & =S^{\prime}+\epsilon U^{\prime \prime} \\
-c S^{\prime}+U S^{\prime}-S U^{\prime} & =\alpha U^{\prime}-\beta S .
\end{aligned}
$$

Integrating (4.3), applying the asymptotic boundary conditions, and eliminating $U^{\prime}$ in (4.4) yields the system

$$
\begin{aligned}
\epsilon U^{\prime} & =\frac{1}{2}\left(U-u_{\ell}\right)\left(U-u_{r}\right)-S \\
\epsilon(U-c) S^{\prime} & =(S+\alpha)\left(\frac{1}{2}\left(U-u_{\ell}\right)\left(U-u_{r}\right)-S\right)-\epsilon \beta S .
\end{aligned}
$$

This system has precisely two equilibrium points $\left(u_{\ell}, 0\right)$ and $\left(u_{r}, 0\right)$. A traveling wave solution of the PDE system (4.1)-(4.2) corresponds to a heteroclinic orbit connecting these two equilibrium points, as in Figure 4.1 (recall we are assuming $u_{\ell}>u_{r}$ ).

Note that if a traveling wave of the original system (1.3)-(1.4) exists (i.e., when $\left.\alpha>\left(u_{\ell}-u_{r}\right)^{2} / 4\right)$, then the wave corresponds to the trajectory in the phase plane defined by (3.5), or equivalently,

$$
S=\frac{1}{2}\left(U-u_{\ell}\right)\left(U-u_{r}\right)
$$

This is the $U$-nullcline from (4.5) (for all $\epsilon$ ).

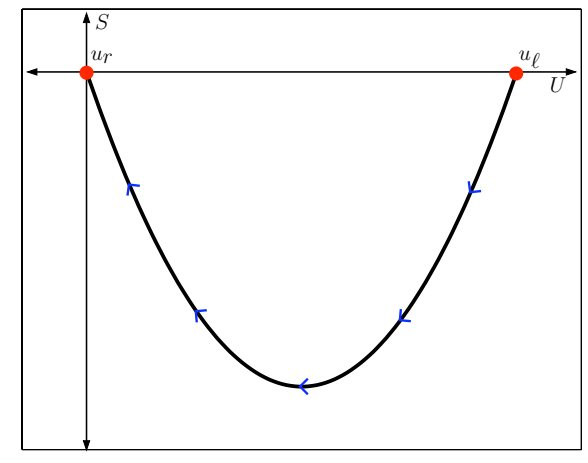

FIG. 4.1. Heteroclinic orbit corresponding to traveling wave solution of system (4.1)-(4.2).

The system (4.5)-(4.6) exhibits symmetric behavior about the line $U=c$, where $c=\left(u_{\ell}+u_{r}\right) / 2$ is the wave speed for the inviscid case $(\epsilon=0)$. In particular, if $(\widehat{U}(\xi), \widehat{S}(\xi))$ solves (4.5)-(4.6) with $\widehat{U}>c$ for $\xi \in\left(-b, \xi_{0}\right)$ and $\widehat{U}\left(\xi_{0}\right)=c$, then $(U(\xi), S(\xi))=\left(2 c-\widehat{U}\left(-\xi+2 \xi_{0}\right), \widehat{S}\left(-\xi+2 \xi_{0}\right)\right)$ solves (4.5)-(4.6) for $\xi \in\left(\xi_{0}, b+2 \xi_{0}\right)$, with $U<c$ and $U\left(\xi_{0}\right)=c$. This corresponds to the reflection of the trajectory through the line $U=c$.

The Jacobian of the system at the equilibrium point $\left(u_{\ell}, 0\right)$ is

$$
J=J\left(u_{\ell}, 0\right)=\left[\begin{array}{cc}
\frac{d}{2 \epsilon} & -\frac{1}{\epsilon} \\
\frac{\alpha}{\epsilon} & -\frac{2(\alpha+\beta \epsilon)}{\epsilon d}
\end{array}\right]
$$

where $d=u_{\ell}-u_{r}$. Since $\operatorname{det}(J)=-\frac{\beta}{\epsilon}<0$, it follows that $\left(u_{\ell}, 0\right)$ is a saddle point for all $\epsilon>0$. Thus the reflection through $U=c$ maps the unstable manifold of 
$\left(u_{\ell}, 0\right)$ to the stable manifold of $\left(u_{r}, 0\right)$. For this reason, to establish the existence of a heteroclinic orbit connecting the two, it suffices to establish that the unstable manifold of $\left(u_{\ell}, 0\right)$ crosses the line $U=c$.

The positive eigenvalue of $J\left(u_{\ell}, 0\right)$ is

$$
\lambda_{\ell}=\frac{1}{4 \epsilon d}\left(d^{2}-4(\alpha+\beta \epsilon)+\sqrt{\left(d^{2}-4(\alpha+\beta \epsilon)\right)^{2}+16 d^{2} \beta \epsilon}\right),
$$

with an associated eigenvector

$$
\boldsymbol{v}_{\ell}=\left[1, \frac{d^{2}+4 \alpha+4 \beta \epsilon-\sqrt{\left(d^{2}+4 \alpha+4 \beta \epsilon\right)^{2}-16 \alpha d^{2}}}{4 d}\right] .
$$

The expansion of $\lambda_{\ell}$ for small $\epsilon$ is

$$
\lambda_{\ell}=\frac{1}{4 \epsilon d}\left(\left(d^{2}-4 \alpha\right)+\left|d^{2}-4 \alpha\right|\right)+O(1) .
$$

Thus,

$$
\alpha>\frac{d^{2}}{4} \quad \text { implies } \quad \lambda_{\ell}=O(1) \text { as } \epsilon \rightarrow 0
$$

and

$$
\alpha<\frac{d^{2}}{4} \quad \text { implies } \quad \lambda_{\ell}=\frac{1}{\epsilon}\left(\frac{d^{2}-4 \alpha}{2 d}\right)+O(1) \text { as } \epsilon \rightarrow 0 .
$$

This transition occurs precisely at the critical $\alpha$ value in equation (3.10), which determines existence of traveling waves of the original system $(\epsilon=0)$. Thus the onset of the solutions containing shocks corresponds to the introduction of a fast dynamic along the unstable manifold of $\left(u_{\ell}, 0\right)$ as $\epsilon \rightarrow 0$. Our motivation for introducing the viscous regularization was to understand the behavior of the wave solutions in the limit of $\epsilon \rightarrow 0$. Accordingly, we now focus on the case $0<\alpha<d^{2} / 4$, the range for which classical traveling waves of the original system $(\epsilon=0)$ fail to exist. There are two cases, depending on whether $0<\alpha<d^{2} / 8$ or $d^{2} / 8<\alpha<d^{2} / 4$.

4.1. Case 1: $d^{2} / 8<\alpha<d^{2} / 4$. The $U$-nullcline is the parabola given by (4.7). There are two distinct nullclines for $S$ which correspond to the solutions of

$$
(S+\alpha)\left(\frac{1}{2}\left(U-u_{\ell}\right)\left(U-u_{r}\right)-S\right)-\epsilon \beta S=0 .
$$

To plot the $S$-nullclines, we arrange equation (4.14) to

$$
(U-c)^{2}=2 S+\frac{d^{2}}{4}+\frac{2 \beta S}{\alpha+S} \epsilon .
$$

When $\epsilon=0$, the curve

$$
(U-c)^{2}=2 S+\frac{d^{2}}{4}
$$

is identical to the $U$-nullcline given by equation (4.7). 
One $S$-nullcline is located above (in the $U$-S plane) the horizontal line $S=-\alpha$, and the other below this line. For $-\alpha<S<0$, the last term in (4.15), $2 \beta S /(\alpha+S)$, is always negative. This decreases $U^{2}$, meaning that there is an $S$-nullcline just above the $U$-nullcline (just below for $S>0$ ). As $\epsilon \rightarrow 0$, this $S$-nullcline converges to the $U$-nullcline.

On the second $S$-nullcline, $S<-\alpha$. In this region, the last term in (4.15) is always positive, and for $S$ close to $-\alpha$ it dominates the linear term. The minimum value of $S$ on the $U$-nullcline is $-d^{2} / 8$. Since $\alpha>d^{2} / 8$, this second $S$-nullcline is below the $U$-nullcline and bounded away from it as $\epsilon \rightarrow 0$. A sample plot of all three nullclines is shown in Figure 4.2.

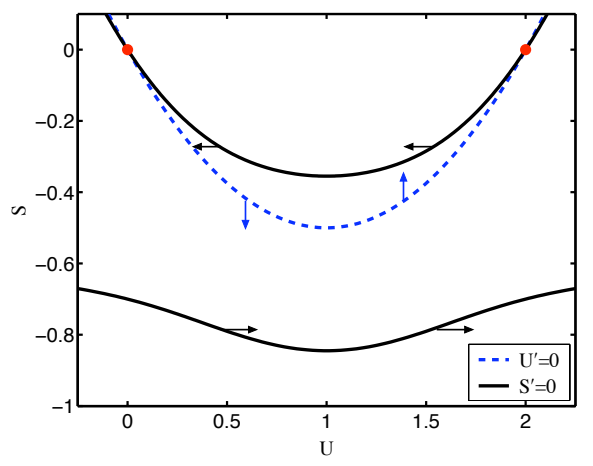

FIG. 4.2. Typical nullclines for system (4.5)-(4.6) with $d^{2} / 8<\alpha<d^{2} / 4$. Here the parameter values are $u_{\ell}=2, u_{r}=0, \beta=1, \alpha=0.6, \epsilon=0.1$.

To find a traveling wave solution, we show that the unstable manifold of $\left(u_{\ell}, 0\right)$ flows to the line $U=c$. The eigenvector $\boldsymbol{v}_{\ell}$ from (4.10) is tangent to the unstable manifold at $\left(u_{\ell}, 0\right)$. Expanding this eigenvector for small $\epsilon$ yields

$$
\boldsymbol{v}_{\ell}=\left[1, \frac{2 \alpha}{d}\right]+\epsilon\left[0, \frac{-8 \alpha \beta}{d\left(d^{2}-4 \alpha\right)}\right]+O\left(\epsilon^{2}\right)
$$

Thus, in the limit as $\epsilon \rightarrow 0$ the eigenpair $\left(\lambda_{\ell}, \boldsymbol{v}_{\ell}\right) \rightarrow(\infty,[1,2 \alpha / d])$. The slope of the $U$-nullcline at $\left(u_{\ell}, 0\right)$ is $d / 2$ (independent of $\epsilon$ ) and the slope of the $S$-nullcline at $\left(u_{\ell}, 0\right)$ is $d / 2(1+\beta \epsilon / \alpha)^{-1}=d / 2(1-\beta \epsilon / \alpha)+O\left(\epsilon^{2}\right)$. Thus, for $\epsilon<\alpha / \beta$, the unstable manifold enters the region above both the $S$ - and $U$-nullclines whenever $\alpha<d^{2} / 4$. Moreover, as $\epsilon \rightarrow 0$ the speed with which it enters the region approaches infinity.

The trajectories of the system (4.5)-(4.6) satisfy

$$
\frac{d S}{d U}=\frac{(S+\alpha) F(U, S)-\epsilon \beta S}{(U-c) F(U, S)}
$$

where $F(U, S)=\frac{1}{2}\left(U-u_{\ell}\right)\left(U-u_{r}\right)-S$. Note that $F(U, S)=0$ defines the $U$-nullcline and is the leading order approximation of the $S$-nullcline above it. The unstable manifold quickly flows away from these nullclines into the region where $F(U, S)=$ $O(1)$. In this case, the curves defined by (4.18) are approximated by

$$
\frac{d S}{d U}=\frac{(S+\alpha)}{(U-c)} .
$$

The solutions of (4.19) are lines of the form $|S+\alpha|=A(U-c)$. The solution passing through the equilibrium $\left(u_{\ell}, 0\right)$ has slope $A=2 \alpha / d$, which is precisely the slope of 
the unstable manifold as $\epsilon \rightarrow 0$. Therefore the leading order approximation to the unstable manifold is

$$
S=\frac{2 \alpha}{d}(U-c)-\alpha,
$$

which is a valid approximation as long this trajectory remains away from the nullclines. The line (4.20) eventually intersects the $S$-nullcline. To leading order, this intersection occurs at

$$
\begin{aligned}
U^{*} & =\frac{4 \alpha}{d}+u_{r}, \\
S^{*} & =\frac{2 \alpha}{d^{2}}\left(4 \alpha-d^{2}\right) .
\end{aligned}
$$

Since $\alpha>d^{2} / 8$, it follows $U^{*}>c$ at the point of intersection. Near the nullclines, the solution to the system (4.5)-(4.6), can be approximated by the quasisteady solution

$$
S=\frac{1}{2}\left(U-u_{\ell}\right)\left(U-u_{r}\right)+O(\epsilon) .
$$

This trajectory intersects the line $U=c$. Thus, by the symmetry of the system, this solution is part of a heteroclinic orbit connecting the points $\left(u_{\ell}, 0\right)$ and $\left(u_{r}, 0\right)$, and corresponds to a traveling wave solution of (4.1)-(4.2).

The above analysis explains the double-shock solution. When $\alpha<d^{2} / 4$, the dynamics near the point $\left(u_{\ell}, 0\right)$ on the unstable manifold are very fast $\left(O\left(\epsilon^{-1}\right)\right)$. Leaving the equilibrium point, the unstable manifold moves away from the nullclines, but eventually this trajectory approaches the nullclines near the point $\left(U^{*}, S^{*}\right)$ away from the equilibrium point. This path in phase space (in the limit $\epsilon \rightarrow 0$ ) corresponds to the shock. Once near the nullclines, the solution flows along the nullclines, to the line $U=c$. The flow between the point $\left(U^{*}, S^{*}\right)$ and its reflected point $\left(2 c-U^{*}, S^{*}\right)$ corresponds to the smooth portion of the double-shock solution between the two shocks. Figure 4.3(a) shows the path of the heteroclinic orbit in phase space corresponding to a double-shock solution. The path shown was generated by integrating (4.5)-(4.6) for $\epsilon=10^{-3}$. The trajectory is very close to our asymptotic solution, which is not shown because it is indistinguishable from the numerical solution on this scale. In Figure 4.3(b) we show the wave profile for decreasing values of $\epsilon$. The solutions were generated by integrating (4.5)-(4.6) for $U>c$ and using the symmetry condition for $U<c$. For finite $\epsilon$ the wave is smooth, but as the figure indicates, the profile approaches the double-shock solution as $\epsilon \rightarrow 0$.

The height of each of the shocks in the double-shock solution is given by

$$
[u]=u_{\ell}-U^{*}=\frac{d^{2}-4 \alpha}{d} .
$$

Below $\alpha=d^{2} / 4$ smooth traveling waves no longer exist, and at this value of $\alpha$ the shock height is zero. As $\alpha$ decreases from this value, the height of the shocks increases. When $\alpha=d^{2} / 8$, the height of each shock is $d / 2$ so that the two shocks come together, and the double-shock solution as analyzed in this section no longer exists. What happens below this value of $\alpha$ is considered in the next section.

4.2. Case 2: $0<\alpha<d^{2} / 8$. Much of the analysis from the previous section applies to this case. However, one exception is that the $S$-nullcline above the $U$ nullcline no longer converges to the $U$-nullcline as $\epsilon \rightarrow 0$. As before, one of the 


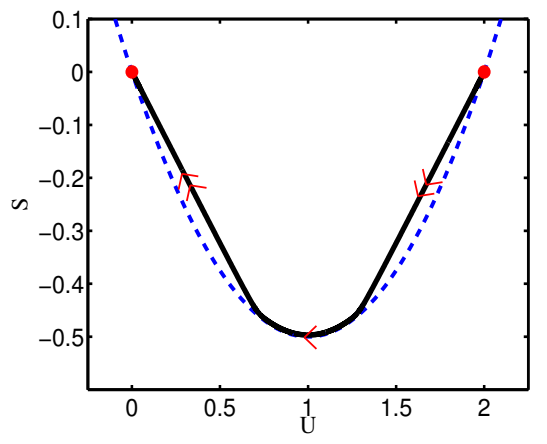

(a)

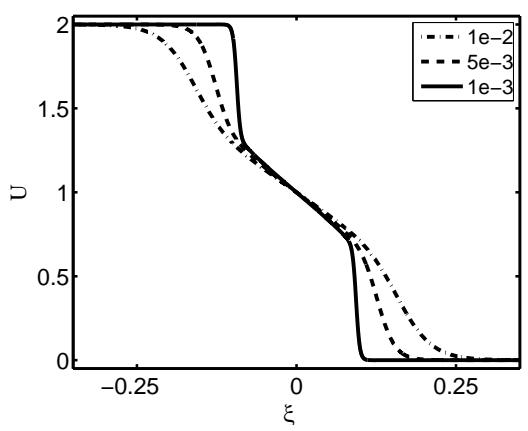

(b)

FIG. 4.3. (a) Path of the heteroclinic orbit for the double-shock traveling wave. The double arrows indicate that the dynamics are much faster along these paths which correspond to the shocks in the limit $\epsilon \rightarrow 0$. The trajectory shown is for $\epsilon=10^{-3}$ generated by integrating (4.5)-(4.6). The solution is indistinguishable from the asymptotic solution on the scale shown. (b) For finite $\epsilon$, the wave profile is smooth, but as $\epsilon \rightarrow 0$, the solution approaches the double-shock wave. The parameter values are $u_{\ell}=2, u_{r}=0, \beta=1, \alpha=0.65$.

$S$-nullclines is located above the line $S=-\alpha$ and the other below. Recall that the $U$-nullcline is the parabola (4.7), and the minimum value of $S$ on this nullcline is $-d^{2} / 8$. When $\alpha<d^{2} / 8$, the line $S=-\alpha$ intersects the $U$-nullcline, so that as $\epsilon \rightarrow 0$ the $S$-nullcline above the $U$-nullcline remains bounded away from the $U$-nullcline for a range of $U$ values. This $S$-nullcline limits to

$$
S= \begin{cases}\frac{1}{2}\left(U-u_{\ell}\right)\left(U-u_{r}\right) & (U-c)^{2}>\frac{d^{2}-4 \alpha}{8} \\ -\alpha & (U-c)^{2} \leq \frac{d^{2}-4 \alpha}{8}\end{cases}
$$

A sample plot of the nullclines is shown in Figure 4.4 for small $\epsilon$.

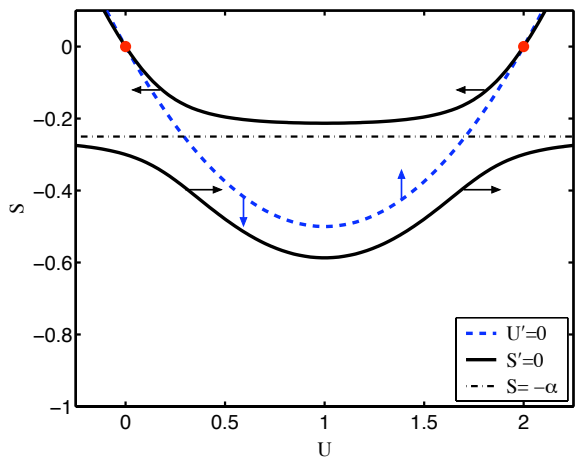

FIG. 4.4. Typical nullclines for system (4.5)-(4.6) with $0<\alpha<d^{2} / 8$. Here the parameter values are $u_{\ell}=2, u_{r}=0, \beta=1, \alpha=0.25, \epsilon=0.05$.

As before, the unstable manifold of $\left(u_{\ell}, 0\right)$ flows into the region above the $S$ nullcline, and once the trajectory is $O(\epsilon)$ away from the equilibrium point the dynamics are fast $\left(O\left(\epsilon^{-1}\right)\right)$. This unstable manifold is again approximated by the line (4.20). The unstable manifold eventually brings the flow back to the $S$-nullcline (4.25). These 
two curves intersect at the point $(U, S)=(c,-\alpha)$, and by symmetry the stable manifold of $\left(u_{r}, 0\right)$ also flows from this point. Thus the solution does not travel along the $S$-nullcline at all because the region of fast dynamics leaving $\left(u_{\ell}, 0\right)$ connects with the region of fast dynamics entering $\left(u_{r}, 0\right)$. Figure 4.5(a) shows the path of the heteroclinic orbit connecting $\left(u_{\ell}, 0\right)$ and $\left(u_{r}, 0\right)$ corresponding to the single-shock traveling wave. This solution was generated by integrating (4.5)-(4.6) for $\epsilon=0.02$. The asymptotic solution is indistinguishable from the numerical solution on this scale. Figure 4.5(b) shows the wave profile for decreasing values of $\epsilon$. For finite $\epsilon$ the wave profile is smooth, but it approaches a single shock as $\epsilon \rightarrow 0$.

The numerical simulations from Section 3.3 suggested that for $\alpha<d^{2} / 8$ the traveling wave solution was the shock solution from the inviscid Burgers' equation. This analysis confirms this, but provides more information on the structure of this shock for small viscosity. This shock is really a degenerate double-shock solution in that the two shocks meet in the middle of the wave profile.

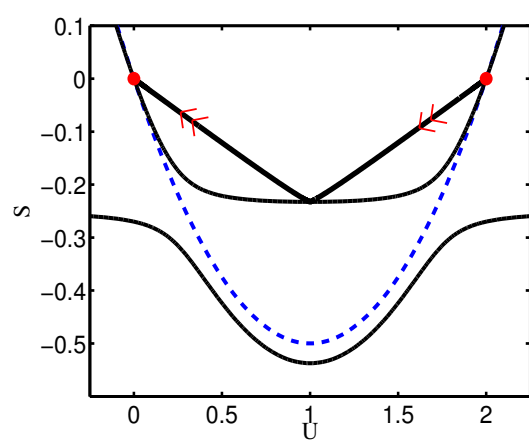

(a)

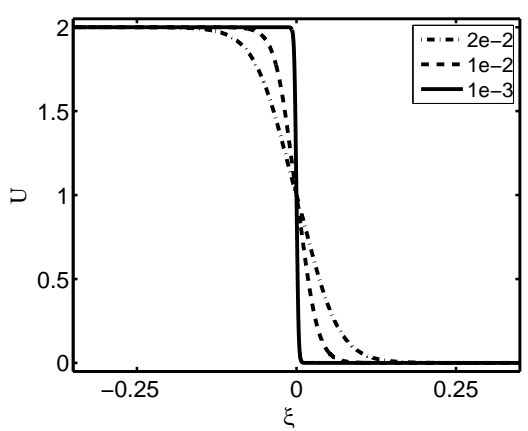

(b)

FIG. 4.5. (a) Path of the heteroclinic orbit for the single shock traveling wave that occurs when $\alpha<d^{2} / 8$. The double arrows indicate the fast dynamics along these paths which correspond to the shocks in the limit $\epsilon \rightarrow 0$. This solution was generated by integrating (4.5)-(4.6) for $\epsilon=0.02$. On this scale the asymptotic solution is indistinguishable from the numerical solution. (b) For finite $\epsilon$ the wave profile is smooth, but as $\epsilon \rightarrow 0$ the solution approaches the single-shock wave. The parameter values in both plots are $u_{\ell}=2, u_{r}=0, \beta=1, \alpha=0.25$.

5. Discussion. For given asymptotic values of the velocity, $u_{\ell}$ and $u_{r}$ with $u_{\ell}>$ $u_{r}$, the viscoelastic Burgers' model (1.3)-(1.4) has three different types of traveling wave solutions, depending on the value of the elastic modulus $\alpha$. For $\alpha>d^{2} / 4$, smooth traveling waves exist, where $d=u_{\ell}-u_{r}$. When $d^{2} / 8<\alpha<d^{2} / 4$, the profile of the traveling wave is piecewise smooth with two jump discontinuities, and when $\alpha<d^{2} / 8$ the wave solution is a single shock. In all three cases the wave travels with unique speed $c=\left(u_{\ell}+u_{r}\right) / 2$.

We address the physical significance of the threshold in the elastic modulus $\alpha$ for traveling waves to exist. For simplicity, consider the case in which $u_{\ell}=-u_{r}$, so that the speed of the traveling wave is 0 . The condition $\alpha>d^{2} / 4$ for a wave to exist reduces to $\alpha>u_{\ell}^{2}$, or $\sqrt{\alpha}>u_{\ell}$. The system linearized about $u=u_{\ell}, \sigma=0$ is

$$
\begin{aligned}
& u_{t}+u_{\ell} u_{x}=\sigma_{x} \\
& \sigma_{t}+u_{\ell} \sigma_{x}=\alpha u_{x}-\beta \sigma,
\end{aligned}
$$


which can be written in the form

$$
\boldsymbol{q}_{t}+A \boldsymbol{q}_{x}=B \boldsymbol{q},
$$

where $\boldsymbol{q}=(u, \sigma)^{\mathrm{T}}$. The wave speeds of this linearized system are $u_{\ell} \pm \sqrt{\alpha}$. The waves speeds are the sum of the advective speed $u_{\ell}$ and the elastic wave speeds $\pm \sqrt{\alpha}$. The advection terms tend to steepen the wave, which generates elastic forces that oppose this steepening. As long as the elastic wave speed is faster than the advective wave speed, smooth traveling waves exist. In the viscous Burgers equation $\left(\sigma=\epsilon u_{x}\right)$, the viscous stresses propagate instantaneously, but in the viscoelastic model the elastic stresses propagate at a finite speed. Thus, the smooth traveling wave breaks down when the advective speed surpasses the elastic speed.

Recall that $\alpha=\mu / \lambda$, where $\mu$ and $\lambda$ are the viscosity and relaxation time, respectively. For a fixed relaxation time, each of the three types of wave solutions is possible, depending on the size of the viscosity. For large enough viscosity, the smooth traveling wave results, and as the viscosity is decreased the solution transitions to the double-shock wave and then to the single-shock wave. Equivalently, for a fixed viscosity, the type of wave depends on the size of the relaxation time. The progression from the smooth wave to the double-shock wave to the single-shock wave occurs as the relaxation time increases. The regions of parameter space where the different wave solutions occur is illustrated in Figure 5.1.

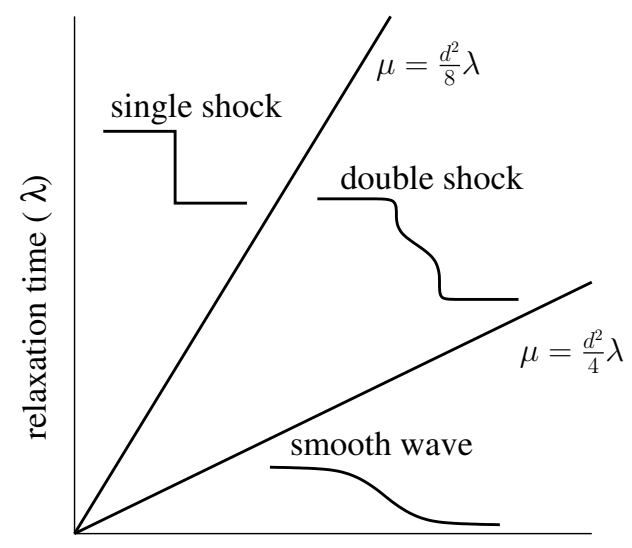

viscosity $(\mu)$

FIG. 5.1. The values of the relaxation time $\lambda$ and the viscosity $\mu$ determine the type of traveling wave solution. In parameter space the line $\mu=d^{2} \lambda / 4$ is the boundary between smooth waves and double-shock solutions, and the line $\mu=d^{2} \lambda / 8$ is the boundary between double shocks and single shocks.

For $\lambda=0$, only the smooth traveling wave is possible. In the limit that $\lambda \rightarrow 0$ for fixed $\mu$, the constitutive law reduces $\sigma=\mu u_{x}$, and the model becomes Burgers' equation (1.1). This limit corresponds to the constitutive law for a viscous fluid. Taking the limit $\beta \rightarrow 0$ for a fixed value of $\alpha$, the constitutive law limits to that of an elastic solid. The transitions between the different wave types are independent of the value of $\beta$. Had we nondimensionalized the problem, the value of $\beta^{-1}=\lambda$ determines the time scale of the problem, which is related to the steepness of the wave profiles. As $\beta$ gets smaller, the wave profiles steepen, meaning that as $\beta \rightarrow 0$, all wave solutions tend to shocks. 
In Section 2, we presented several different one-dimensional reductions of the UCM equation, and in the remainder of the paper presented an analysis based on equation (2.10). However, the techniques employed apply to all three constitutive laws. Repeating the analysis for (2.11), we find that again there are smooth traveling waves for $\alpha>d^{2} / 4$, but for $\alpha<d^{2} / 4$, only the single-shock solutions occur. For (2.12) there is a transition from a smooth traveling wave to a double-shock solution at $\alpha=c^{2}$, and the single shock solution is approached as $\alpha \rightarrow 0$. Because (2.10) exhibits all three behaviors, we chose to present this case.

There are many different constitutive laws for viscoelastic fluids. In this paper we used the UCM model (Oldroyd-B when $\epsilon \neq 0$ ) because it is perhaps the simplest differential constitutive law and it has been extensively studied in the past. Others have studied viscoelastic generalization of Burgers' equation [13, 21], and it would be interesting to explore how the behavior of the wave solutions analyzed in this paper are affected by different constitutive laws.

The problem in this paper is interesting in part because of its classical nature, but the analysis of one-dimensional waves in viscoelastic generalizations of Burgers' equations could also be used to develop numerical schemes for viscoelastic fluids. High-resolution finite-volume methods have been used successfully in simulating high Reynolds number flows [1]. The algorithm for discretizing the convection terms in [1] is based on numerical methods for conservation laws [6]. These methods require solving one-dimensional Riemann problems, and it is not clear how to adapt this approach to nonconservative systems. Wave propagation algorithms [15] are more easily adapted to nonconservative problems, but these methods also require being able to solve one-dimensional Riemann problems. Recently finite-volume methods for viscoelastic flows have been proposed [10, 22]. The techniques from this paper could be adapted to solve the Riemann problems that arise in these methods.

Acknowledgements. The authors would like to thank Andy Bernoff and Jim Keener for helpful discussions while writing this paper. We also thank the referees for their insightful comments.

\section{REFERENCES}

[1] J. B. Bell, P. Colella, and H. M. Glaz, A second-order projection method for the incompressible Navier-Stokes equations, J. Comput. Phys., 85 (1989), pp. 257-283.

[2] H. A. Biagioni, A nonlinear theory of generalized functions, vol. 1421 of Lecture Notes in Mathematics, Springer-Verlag, Berlin, second ed., 1990.

[3] R. B. Bird, R. C. Armstrong, and O. Hassager, Dynamics of Polymeric Liquids, vol. 1, Wiley, New York, 2nd ed., 1987.

[4] - Dynamics of Polymeric Liquids, vol. 2, Wiley, New York, 2nd ed., 1987.

[5] J. M. Burgers, A mathematical model illustrating the theory of turbulence, Adv. Appl. Mech., 1 (1948).

[6] P. Colella, Multidimensional upwind methods for hyperbolic conservation laws, J. Comput. Phys., 87 (1990), pp. 171-200.

[7] J.-F. Colombeau, New generalized functions and multiplication of distributions, vol. 84 of North-Holland Mathematics Studies, North-Holland Publishing Co., Amsterdam, 1984. , Notas de Matemática [Mathematical Notes], 90.

[8] J.-F. Colombeau, Multiplication of Distributions, Lecture Notes in Mathematics, SpringerVerlag, Berlin, 1992.

[9] L. C. Evans, Partial differential equations, vol. 19 of Graduate Studies in Mathematics, American Mathematical Society, Providence, RI, 1998.

[10] R. D. Guy And A. L. Fogelson, A wave propagation algorithm for viscoelastic fluids with spatially and temporally varying properties, Comput. Methods Appl. Mech. Eng., (2007). In Press. 
[11] D. D. Joseph, Fluid Dynamics of Viscoelastic Liquids, Springer, New York, 1990.

[12] R. G. Larson, Constitutive Equations for Polymer Melts and Solutions, Butterworth, Stoneham, MA, 1988.

[13] D. G. Lasseigne And W. E. Olmstead, Stability of a viscoelastic Burgers flow, SIAM J. Appl. Math., 50 (1990), pp. 352-360.

[14] R. J. LeVeque, Numerical Methods for Conservation Laws, Lectures in Mathematics ETH Zürich, Birkhäuser Verlag, Basel, second ed., 1992.

[15] — Finite Volume Methods for Hyperbolic Problems, Cambridge University Press, Cambridge, 2002.

[16] - Finite-volume methods for non-linear elasticity in heterogeneous media, Int. J. Numer. Meth. Fluids, 40 (2002), pp. 93-104.

[17] M. J. Lighthill and G. B. Whitham, On kinematic waves. II. A theory of traffic flow on long crowded roads, Proc. Roy. Soc. London. Ser. A., 229 (1955), pp. 317-345.

[18] J.D. LogAn, An introduction to nonlinear partial differential equations, Pure and Applied Mathematics (New York), John Wiley \& Sons Inc., New York, 1994. , A Wiley-Interscience Publication.

[19] P. I. Richards, Shock waves on the highway, Operations Res., 4 (1956), pp. 42-51.

[20] J. J. Stoken, Water waves, Wiley Classics Library, John Wiley \& Sons Inc., New York, 1992. The mathematical theory with applications, Reprint of the 1957 original, A WileyInterscience Publication.

[21] N. Sugimoto and T. Kakutani, "Generalized Burgers equation" for nonlinear viscoelastic waves, Wave Motion, 7 (1985), pp. 447-458.

[22] D. Trebotich, P. Colella, G. H. Miller, A. Nonaka, T. Marshall, S. Gulati, and D. Liepmann, A numerical algorithm for complex biological flow in irregular microdevice geometries, technical report UCRL-JC-150969, Lawrence Livermore National Laboratory, 2004.

[23] G. B. Whitham, Linear and nonlinear waves, Pure and Applied Mathematics (New York), John Wiley \& Sons Inc., New York, 1999. Reprint of the 1974 original, A Wiley-Interscience Publication. 